\title{
Las PYMEs lideradas por mujeres y la capacitación: un estudio exploratorio
}

\section{SMEs led by women and training: an exploratory study}

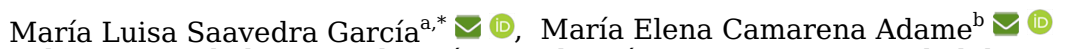

$a, b)$ Universidad Nacional Autónoma de México: Coyoacan, Ciudad de Mexico (México)

* Contacto principal: maluisasaavedra@yahoo.com (María Luisa Saavedra García)

\section{Resumen}

El objetivo de esta investigación consiste en caracterizar la capacitación en las empresas lideradas por mujeres y determinar si existe relación con las características de la empresa y la empresaria. Para ello, se aplicó un cuestionario directo estructurado a una muestra de 272 empresarias de la Ciudad de México. Los principales hallazgos del análisis exploratorio realizado muestran que la mayoría de las empresarias consideran importante la capacitación, sin embargo sólo la mitad se han capacitado, siendo el promedio anual de horas de capacitación 45. Además, únicamente una tercera parte ha implementado programas de capacitación para sus empleados y las acciones de capacitación han sido principalmente en áreas operativas o de producción. Se ha encontrado también una relación significativa entre algunas características de las empresas y empresarias y la capacitación

Palabras clave: capacitación; empresarias; PYMEs; capital humano Clasificación JEL: J16; J24; L26; M53

\begin{abstract}
The objective of this research was to characterize the training in companies led by women and determine if there is a relationship with the characteristics of the company and the businesswoman. A structured direct questionnaire was applied to a sample of 272 voluntary businesswomen from Mexico City. The main findings from this exploratory study show that most women entrepreneurs consider training important; nevertheless only half have been trained, the average annual training hours being 45 . In addition, only one third have implemented training programs for their employees, and the training topics have been mainly in operational or production areas. It was also found a significant relationship between some characteristics of companies and entrepreneurs and training
\end{abstract}

Keywords: training; businesswomen; SMEs; human capital

JEL Classification: J16; J24; L26; M53 


\section{Introducción}

Todas las sociedades le asignan tareas diferentes al hombre y a la mujer (lo que suele llamarse división del trabajo por sexo), es así como se considera que al hombre le corresponde el trabajo productivo y a la mujer el trabajo reproductivo; el trabajo productivo figura en los sistemas de contabilidad nacional, se cuantifica, se reconoce y se remunera; el trabajo reproductivo no se registra en ningún lado, no se cuantifica, no se reconoce, no se remunera, invisibilizando así la labor de la mujer (Daeren, 2000; IICA, 1996).

Sin embargo, se considera que el empoderamiento de la mujer determina el progreso de una nación, en especial el empoderamiento económico, una forma de lograrlo es a través del emprendimiento (Jena, 2018; Santhi, 2017; Tripathi \& Singh, 2018). Así pues, el empoderamiento de la mujer a través del emprendimiento es imprescindible para alcanzar la agenda 2030 y erradicar la pobreza (Deka, 2018). En lo que se refiere al papel de la mujer como empresaria De Vita, Mari, y Poggesi (2014), señalan la importancia del rol que estas juegan no solo en el campo económico sino también social, así pues, el papel de las mujeres en la creación y gestión de empresas es un elemento fundamental e impulsor del crecimiento económico y desarrollo de toda la comunidad; socialmente, la opción de emprendimiento no solo permite a individuos y hogares escapar de la pobreza, sino que también puede ayudar a explorar el poder emancipador de hacer negocios, permitiéndole a la mujer definir su propia identidad a través de logros profesionales.

El capital humano es importante en el desarrollo de las empresas para lograr mejores resultados, por lo que las mujeres empresarias deben tomar en cuenta este recurso tan valioso y preocuparse en capacitarlo y adiestrarlo, con el fin de que esté preparado para asumir los retos que exige el contexto actual de los negocios.

La capacitación de la mujer en áreas identificadas tradicionalmente como masculinas, no sólo puede ampliar las posibilidades de empleo sino también romper la segregación ocupacional existente aboliendo la división del trabajo por sexo, es necesario señalar que esta segregación ocupaciones procede a su vez de una segregación educativa (CEPAL, 2019; Hillmert, 2015; IICA, 1996). En los últimos años, muchas mujeres asisten a cursos para adquirir conocimientos de tareas que están generalmente designadas a hombres, con el fin de mejorar sus ingresos y alcanzar un mejor desarrollo personal (SENCE, 2013).

En este sentido, Abramo, Valenzuela, y Pollack (2000) señalan que la capacitación tiene un impacto importante sobre la productividad e ingreso de la fuerza de trabajo (el retorno promedio de la capacitación en términos de ingreso es $24 \%$, lo que supera el de la educación que es 13\%). Las mujeres experimentan un retorno superior a los hombres al ser capacitadas (ellas obtienen un incremento salarial de 33\% y ellos de 24\%). Sin embargo, el retorno de la educación es mayor para los hombres, por otro lado, las mujeres necesitan credenciales educativas muy superiores a las de los hombres para ingresar al mercado laboral, pero una vez que ingresa, la capacitación le ayuda a mejorar sus credenciales, lo que se traduce en un aumento de ingreso; por su parte, Welsh, Kaciak, y Shamah (2018) encontraron una relación positiva entre capital humano (habilidades de gestión, nivel educativo y edad) y desempeño empresarial en empresas dirigidas por mujeres. A pesar de esto, Todaro, Godoy, y Abramo (2002) encontraron que la percepción de los empresarios es que los costos laborales son más elevados en las mujeres que en los hombres; no obstante, las mujeres resultan mejor evaluadas que los hombres en su desempeño laboral; pudiendo con esto, de algún modo compensar los costos más elevados con un mejor desempeño.

La capacitación se concentra en los trabajadores de empresas grandes, lo que implica una limitación a los aumentos de productividad de las pequeñas y medianas empresas, que es el sector donde se concentran las mujeres. La capacitación con mayor retorno para los hombres es la capacitación en negocios, mientras para las mujeres es la capacitación en salud (seguida también por la capacitación en negocios), lo cual es un indicativo de la segmentación por género del mercado laboral (alta concentración de mujeres como enfermeras y auxiliares de los servicios de salud) y de las dificultades para romper esa segmentación (Abramo et al., 2000; CEPAL, 2019; Tverdostup \& Paas, 2017).

En cuanto a la mujer empresaria, de acuerdo con OIT (2008), el que las mujeres sean propietarias de empresas contribuye de modo importante con el bienestar económico de las familias y las comunidades, a la reducción de la pobreza y al empoderamiento de la mujer. Así también SELA (2010) señala que las mujeres emprendedoras constituyen un componente clave de la economía de los países y un apoyo fundamental al crecimiento económico de países de ingreso medio-alto, particularmente en la región de América Latina y el Caribe, por lo que las mujeres empresarias deben tener un lugar prioritario en el escenario de las políticas públicas, dada la capacidad que tienen para contribuir a crear oportunidades de empleo, emprendimientos y riqueza.

Reforzando lo anterior, los estudios de ONU-Mujeres (2016) han encontrado que existe una correlación positiva entre la actividad empresarial femenina y el crecimiento del Producto Interno Bruto, estima que la productividad podría aumentar un $25 \%$ si se eliminan las barreras al emprendimiento de las mujeres. Sin embargo, no es solo la contribución económica de las empresarias la que merece la importancia de sus empresas, sino que también estas perciben diferentes oportunidades de negocio, ofrecen a la sociedad formas 
nuevas de gestionar las empresas y buscan soluciones alternativas a los problemas que enfrentan durante su ciclo de vida; son capaces de ampliar y diversificar los productos, procesos, forma de organización y mercados objetivos, ofreciendo a los consumidores mejores posibilidades de ajustar sus elecciones a sus preferencias o necesidades (Guzmán \& Rodríguez, 2008; Santhi, 2017). Por su parte, Kray y Kennedy (2017) refieren que cada vez es más aceptado en el mundo de los negocios las habilidades de las mujeres para construir relaciones y evaluar riesgos, lo que a su vez conlleva la permanecía de las empresas. En referencia al riesgo que es capaz de asumir la empresaria GEM (2019) señala que 69\% de las mujeres refieren que no le molesta el miedo al fracaso, contra el $72 \%$ de los hombres.

Sin embargo, a pesar de la gran importancia que tienen las empresas dirigidas por mujeres, algunas de las dificultades que experimentan las empresarias son la falta de experiencia empresarial y de comercialización, insuficiente formación empresarial específica (administración/marketing), escasa actualización e información en temas económicos y financieros, barreras en el acceso a fuentes financieras, barreras en el acceso a redes de comercialización, falta de confianza en sí misma y obstáculos que tienen relación con los prejuicios sociales vinculados al "ser mujer" (Camarena Adame \& Saavedra García, 2016; Daeren, 2000; Heller, 2010). Enfatizando esta problemática las mujeres empresarias del sector PYME han señalado como un obstáculo importante para el desarrollo de sus negocios la falta de capacitación para la administración y dirección y apoyo administrativo y técnico (Cho, Moon, \& Bounkhong, 2019; Zabludovsky, 2001), por lo que resulta relevante realizar estudios en este tópico.

Considerando todo lo anterior, en este estudio de carácter exploratorio se pretende analizar y delimitar las principales características de la capacitación en las empresas lideradas por mujeres así como establecer las relaciones de dicha capacitación con el perfil de las empresarias y de las propias empresas lideradas por mujeres. Para ello, se realizó un estudio en una muestra de 272 empresarias de la Ciudad de México cuyas respuestas y valoraciones al cuestionario estructurado han servido de base para extraer un conjunto de hallazgos sobre la importancia de la capacitación en el contexto de la mujer empresaria.

El trabajo se divide en cuatro secciones. Tras esta introducción, en primer lugar, se aborda la revisión de los principales estudios y hallazgos sobre la capacitación en las empresas dirigidas por mujeres, las características de las mujeres empresarias y las características de las empresas dirigidas por mujeres. En segundo lugar, se describe la metodología del estudio empírico detallando los aspectos de la muestra, el cuestionario y las variables seleccionadas. En tercer lugar, se presentan los resultados del análisis y la interpretación de los mismos para, finalmente, exponer las principales conclusiones de este estudio.

\section{La capacitación en las empresas dirigidas por mujeres}

La importancia de la capacitación, de acuerdo con Attorresi (2009) radica en que por medio de la capacitación es posible generar valor en todos los niveles, económico, laboral, humano, debido a que la capacitación tiene efectos en la sociedad, pues genera conocimiento no sólo para el trabajo sino para la vida mejorando la calidad de ésta. Por otra parte, mediante una capacitación bien estructurada es posible impactar el desempeño de las empresas al modificar la forma en que estas son administradas (Bloom, Eifert, Mahajan, McKenzie, \& Roberts, 2011).

A pesar de lo anterior, el nivel de capacitación de las mujeres empresarias en el sector informal es menor que el de los hombres. Sin embargo, la gran mayoría de los emprendedores en América Latina y el Caribe no cuentan con educación formal, entrenamiento o capacitación empresarial en ningún ciclo escolar o mediante alguna actividad práctica en el ámbito empresarial. Las mujeres con educación formal en América Latina y el Caribe (ALC) que son propietarias de negocios en fases incipientes y negocios establecidos, pero no tienen una educación secundaria son 34.1 por ciento y 40.4 por ciento, respectivamente; es decir que las mujeres con menores niveles de educación tienden a ser más emprendedoras (negocios incipientes) lo que influye en el tipo de empresas que se establecen sobre todo en el sector informal y sus posibilidades de crecimiento, sustentabilidad y desarrollo en el tiempo. Por otra parte, las empresarias en el sector formal y con empresas más grandes y mejor establecidas cuentan con mayores niveles de educación (SELA, 2010).

Coincidente con lo anterior, los estudios realizados por el Banco Mundial (2010) refieren que las empresarias consideran que la capacitación es muy importante y que sería de gran beneficio contar con la capacitación adecuada para impulsar sus empresas. Este estudio también resalta que las empresarias suelen tener niveles considerablemente menores de educación y capacitación que los hombres que poseen un negocio, lo cual puede limitar la productividad y el crecimiento de su negocio, aunque podría reflejar el hecho de que no hay diferencia de género en la opción de hombres y mujeres de convertirse en empresario/a, en contraste con la selección no aleatoria que tiene lugar en el mercado laboral en general, donde la discriminación por género es más visible (Tverdostup \& Paas, 2017). También, señala que si bien las propietarias de empresas no tienen menos habilidades empresariales que los hombres, pero si tienen menos probabilidades de conseguir capacitación y servicios de desarrollo empresarial y cuando existe capacitación para ellas en el mercado, normalmente se concentra en los roles tradicionales (cocina, costura, tejidos, etc.) y excluyéndola de los sectores más productivos y de mayor crecimiento; como consecuencia las empresarias a diferencia de los 
empresarios, tienen capital humano más bajo (en algunos países), por la falta capacitación de calidad y cursos pertinentes.

Así también en India, Santhi (2017) da cuenta de que las mujeres empresarias se enfrentan a la falta de conocimiento sobre como dirigir una empresa, cómo gestionar un préstamo, cómo lidiar con las responsabilidades familiares para comercializar sus productos, desconocimiento de los cambios tecnológicos, por esta razón en cuanto a la obtención de conocimientos por parte de las mujeres sugiere que estos deben enfocarse en temas de gestión, liderazgo, marketing, finanzas, tecnología, proceso de producción, planeación de utilidades, etc. En cierta forma esta falta de conocimiento representa un obstáculo para gestionar la empresa, donde lo que necesita es recibir entrenamiento es decir no solo los conocimientos sino también el know how (Brixiová, Kangoye, \& Said, 2020; Pérez-Pérez \& Avilés-Hernández, 2016).

En referencia a la educación de las mujeres en México, al analizar los grados aprobados en educación superior de 2015 (ANUIES, 2018; SEP, 2019), se observa que en el caso de los estudios de Licenciatura los hombres superan a las mujeres, no así en posgrado dónde las mujeres tienen una mayor participación que los hombres, lo cual resulta relevante pues la educación terciaria se relaciona con un mayor rendimiento empresarial en las empresas de mujeres (Brixiová et al., 2020). En el caso de los estudios de orientados a la carrera educativa predominan con $75 \%$ las mujeres en comparación con el $25 \%$ de los hombres (SEP, 2019). En lo que se refiere a las carreras técnicas son ámbitos donde las mujeres sufren discriminación y acoso por lo que muchas abandonan los estudios (Buquet \& Moreno, 2011).

Asimismo, existen algunas diferencias y similitudes respecto a la elección de carreras universitarias, entre los estudiantes del sexo femenino y masculino. Entre las profesiones más demandadas por ambos sexos se encuentran: Derecho, Administración, Psicología, Ingeniería Industrial y Administración de empresas; generalmente, las mujeres prefieren las carreras de enfermería, educación preescolar, contaduría y educación Primaria (INEGI, 2018) mientras que los hombres se orientan más a la ingeniería, manufactura u construcción, así como sistemas computacionales, agronomía y veterinaria).

Lo anterior denota que la tendencia de estudios por parte de la mujer en México, sigue siendo hacia las áreas que tradicionalmente se ven como una extensión de las labores domésticas y de cuidado; por lo que se hace necesario revertir esta tendencia para lograr el empoderamiento económico de las mujeres en áreas de rentabilidad elevada. Las mujeres se encuentran relegadas de áreas de estudios conocidas como STEM (por sus siglas en inglés ciencia, tecnología, ingeniería y matemáticas) la cual se observa a lo largo de todo el proceso educativo, donde se ven orilladas hacia las áreas de enseñanza, salud y asistencia social, actividades que generan menores ingresos (CEPAL, 2019).

En lo que se refiere a la formación de la empresaria, Correia Santos (2015) encontraron en España que la mujer tiene menos formación emprendedora (33.9\% para hombres frente al 27.9\% para mujeres), sin embargo, en las mujeres que se encuentran en la etapa inicial de una actividad emprendedora se observa una formación específica en emprendimiento superior a la de los emprendedores $(44.2 \%$ de emprendedores frente al $46.7 \%$ de emprendedoras). Se observa también, un mayor número de emprendedoras con titulación universitaria ( $35 \%$ con titulación media, superior, máster o doctorado frente a un $24.7 \%$ de hombres). En cuanto a su especialización educativa, se advierte un incremento en la diversidad de carreras desarrolladas por parte de las mujeres emprendedoras (48.6\% en ciencias sociales y económicas, $9.2 \%$ en técnicas, $2.6 \%$ en arte, $5.2 \%$ en ciencia, $11.8 \%$ en ciencias de la salud y $22.6 \%$ en humanidades). Así también, los hombres cuentan con más años de experiencia laboral que las mujeres.

En el caso de las mujeres empresarias mexicanas su principal base formativa se compone de conocimiento empírico adquirido en sus tareas dentro de las empresas familiares y en menor cantidad de conocimiento técnico e instrumental y se ha demostrado que el conocimiento empresarial heredado, es importante para asegurar el éxito de los negocios de las mujeres (Lopez Sullivan, 2013). Coincidiendo así con Inmujeres (2009) en el sentido de que entre los principales problemas que enfrentan las empresas dirigidas por mujeres en México se encuentra la falta de capacitación técnica. Esto resulta preocupante si se considera que Tripathi y Singh (2018) y Brixiová et al. (2020) señalan que esta falta de habilidades técnicas limita la entrada a sectores de mayor valor agregado o su aumento de la productividad en las empresas dirigidas por mujeres.

La importancia de la capacitación de las mujeres empresarias radica en que estas se sienten menos capacitadas y temen al fracaso más que los hombres, por lo que la capacitación puede reducir esta desventaja y brindarle la confianza y habilidades necesarias para gestionar su empresa (Brixiová et al., 2020; Jena, 2018; Tripathi \& Singh, 2018). En este mismo sentido, GEM (2019) señala que las empresarias se perciben con menos habilidades (74\%) para dirigir una empresa que los hombres (84\%), lo cual de por si constituye un obstáculo para llevar a cabo sus planes empresariales. Por su parte Brixiová et al. (2020) afirman que las mujeres pueden estar rezagadas con respecto a los hombres en habilidades blandas, como iniciativa propia, innovación, identificación y explotación de nuevas oportunidades, establecimiento de objetivos, planeación y superación de obstáculos. 
En cuanto a los efectos de la capacitación, en las empresas dirigidas por mujeres, Valdivia (2014) en una investigación empírica que tuvo como objetivo evaluar el impacto de la capacitación en las empresas dirigidas por mujeres, usando una muestra de 2,400 mujeres empresarias de Perú, encuentra que las microempresarias que participaron en el estudio, unos dos años después de haber terminado la capacitación experimentaron un incremento en sus ventas de más de 15\%; por lo que concluyen que ofrecer asistencia técnica acompañada de capacitación tiene como efecto un crecimiento rápido en el corto plazo. Coincidiendo con el estudio de Sapién Aguilar, Piñón Howlet, y Gutiérrez Díez (2014) quienes señalan que las actividades de capacitación se consideran generadoras de aumento en la competitividad y productividad de las medianas empresas.

En otra investigación, Intersocial (2011) comprobó que con una estrategia de capacitación integral a las mujeres empresarias, son capaces de asimilar mejor los conocimientos, puesto que el resultado fue que el $64 \%$ de las mujeres capacitadas aplicaron los conocimientos que obtuvieron con la capacitación, implementado y/o mejorando sus iniciativas de negocios, demostrando sus habilidades productivas (en la elaboración de diferentes productos con procesos estandarizados de producción y mayor calidad en el acabado del producto) y capacidad de gestión empresarial, mejorando la administración y contabilidad de su negocio, aplicando herramientas de marketing y ampliando la búsqueda de contactos comerciales para la venta de sus productos; como consecuencia de estas acciones el $64 \%$ mejoraron sus ingresos.

\subsection{Caracterización de mujeres empresarias y de empresas dirigidas por mujeres}

\subsubsection{Perfil y características de las mujeres empresarias}

De acuerdo con Guzmán y Rodríguez (2008), Banco Mundial (2010) y OIT (2015) las mujeres emprenden motivadas por el autoempleo y la existencia de techos de cristal $^{1}$ (motivos extrínsecos), la necesidad de logro, autorrealización, deseo de independencia (motivos intrínsecos), el deseo de compaginar tareas empresariales con familia y hogar (motivos trascendentales), necesidad de complementar los ingresos familiares o mantener totalmente a la familia (motivos económicos); presentan un menor grado de formación empresarial y experiencia laboral, y pocas han ocupado puestos directivos, en comparación con los varones. Las empresarias también tienden a administrar sus negocios desde su hogar; disminuyendo costos y riesgos y aumentando la flexibilidad para compaginar sus actividades con las labores del hogar. Es importante señalar que la base formativa de las mujeres sobre los negocios se compone de conocimiento empírico adquirido en sus tareas dentro de empresas familiares y en menor cantidad de conocimiento técnico e instrumental; se ha demostrado que el conocimiento empresarial heredado es importante para asegurar el éxito de los negocios de las mujeres (Lopez Sullivan, 2013).

Por su parte, Heller (2010) y De Vita et al. (2014) señalan que las mujeres en Latinoamérica emprenden principalmente como una alternativa de autoempleo convirtiéndose en muchos casos en una estrategia de supervivencia, especialmente en periodos de crisis y desempleo; otras lo hacen con el fin de llevar a cabo una idea innovadora y otras más por alcanzar un nicho específico en el mercado. En el caso de las mujeres profesionales que deciden emprender al no poder avanzar en sus carreras profesionales debido al "techo de cristal" y/o a la necesidad de equilibrar su vida laboral con la familiar, tienen como característica común que cuentan con un nivel de estudios mayor al de los empresarios varones.

Las mujeres empresarias Latinoamericanas, son de edad media mayor de 35 años, casadas y con hijos (aunque existe una proporción alta de mujeres separadas), cuentan con altos niveles educativos, dedican más de 48 horas semanales a la actividad empresarial y encuentran satisfacción en su trabajo puesto que les permite realizarse profesionalmente y autoafirmarse (Daeren, 2000). En estudios posteriores, Heller (2010) y De Vita et al. (2014) encuentran que las mujeres emprendedoras en esta región tienen entre 31 y 45 años y en su mayoría están casadas y con hijos pequeños, mostrando así que estas características demográficas de las empresarias no han cambiado.

Santhi (2017), en una muestra de 100 empresarias en India, encontró que predominaban las mujeres de entre 36 y 45 años, las cuales formaron su empresa ya casadas, en cuanto al nivel de estudios el $26 \%$ no están graduadas, el $31 \%$ son graduadas y el $43 \%$ son postgraduadas. El $75 \%$ manifestó haber recibido apoyo de su familia para emprender y también el $75 \%$ pertenecen a una familia nuclear, es decir se encuentran casadas y con hijos.

Por su parte, Inmujeres (2005) e Inmujeres (2009) han encontrado que en México las mujeres emprenden principalmente por la necesidad de mejorar su calidad de vida y obtener ingresos propios, así como por la búsqueda de independencia y el deseo de un negocio propio; el $42 \%$ de las empresarias manifestaron que su padre había sido o era empresario, el 27\% el cónyuge y el 23\% la madre, sin embargo, el 27\% no tiene antecedente alguno de actividad empresarial. Asimismo, se encontró que el 87\% de las empresarias contaban con antecedentes laborales antes de iniciar sus empresas; en los que se refiere a las características demográficas, el 33\% tenían entre 34 y 55 años de edad, el 25\% eran menores de 35 años y el $15.5 \%$ eran mayores de 55 años; la mayoría de las mujeres vivían en unión libre y sólo el 25\% se encontraban casadas; el $47.9 \%$ contaba con estudios de licenciatura y el $28.7 \%$ con estudios de posgrado, la mujeres incrementaron su 
nivel de estudios ante la necesidad de contar con mayores conocimientos para llevar a cabo su actividad empresarial.

Asimismo, en México, Zabludovsky (2001) encontraron que las mujeres se encontraban mejor preparadas en habilidades interpersonales y relaciones con los clientes y menos preparadas en habilidades técnicas y gerenciales y para asumir riesgos, coincidiendo con lo señalado por Deka (2018) y Tripathi y Singh (2018) acerca de la falta de limitada capacidad de gestión de las mujeres lo que no les permite llevar con éxito su empresa. Hernández (2015), con base en otro estudio, señala que las mujeres trasladan los valores domésticos a la empresa, así pues, incorporan rasgos como la paciencia, la protección y el interés por los demás, considerados típicamente femeninos.

Elizundia Cisneros (2015), por su parte, encuentra que en México no existen diferencias significativas entre el nivel de estudios de las emprendedoras en comparación con los emprendedores, en el nivel superior de estudios, sin embargo, en el nivel de posgrado son más los emprendedores los que alcanzan este, sin llegar a ser significativa la diferencia.

Value for Women (2018), al investigar a las mujeres empresarias en México, encontró que, en el segmento de empresas en crecimiento, las empresarias en su mayoría tienen grado de licenciatura o de posgrado y una amplia experiencia laboral. La gran mayoría refiere que reciben apoyo de la familia o cónyuge para sus emprendimientos (92\%). Sin embargo, también manifiestan que la carga de la crianza de los hijos y labores del hogar sigue recayendo en gran parte sobre ellas, lo que reduce significativamente el tiempo que le deben dedicar a la empresa y a su propia capacitación.

GEM (2019) encuentra que las tasas más altas de participación en el emprendimiento empresarial entre las mujeres en el mundo, se encuentran en los grupos de edad de 25 a 34 y de 35 a 44 años, estas tasas aumentan con el nivel de educación, sin embargo la educación de nivel posgrado disminuye en comparación con los hombres, excepto en África donde las mujeres con este nivel de estudios son más activas; el 63\% son capaces de percibir oportunidades de emprendimiento, destacándose las de países menos desarrollados. Asimismo, este estudio señala que el $34 \%$ de las mujeres emprendedoras trabaja sola sin socios ni empleados, en comparación con el $26 \%$ de los hombres emprendedores.

En referencia a la capacitación de las empresarias, Value for Women (2018) señala que las empresarias opinan que el problema es que la información en la mayoría de los cursos es muy básica y poco aplicada a la práctica, necesitando acciones más especializadas y focalizadas en el crecimiento empresarial. Los cursos de mayor calidad y más especializados son costosos. También Espino (2018) se refiere a que los cursos no se encuentran adaptados a las verdaderas necesidades de las mujeres y que es necesaria una capacitación con enfoque de género.

\subsubsection{Características de las empresas dirigidas por mujeres}

Las empresas dirigidas por mujeres se caracterizan por tener menos de 10 años de antigüedad, predominando el tamaño micro y pequeño, operar principalmente en el sector comercial y servicios (Daeren, 2000), sectores en los que se necesita menos capital financiero, humano e infraestructura para operar (Heller, 2010).

Las empresas propiedad de mujeres en comparación con aquellas cuyo dueño es un hombre, en general, usan menos mano de obra y capital físico y, en algunos países, registran niveles más bajos de capital humano, repercutiendo esto tanto en la productividad como en la rentabilidad (Banco Mundial, 2010). También, suelen concentrarse en un menor número de sectores económicos, predominando el sector comercio, quedándose en su mayoría en una etapa incipiente de su negocio, tal vez por la aversión al riesgo; estas características se encuentran sobre todo en la pequeña y la microempresa y muy pocas están en empresas grandes o medianas y (Cho et al., 2019; Heller, 2010; Saavedra García \& Camarena Adame, 2015).

Según SELA (2010) y Berger y Kuckertz (2016) las PYMES de mujeres son distintas a las PYMES de hombres, surgen por razones diferentes y sus necesidades son diferentes pues, hombres y mujeres operan de forma distinta, establecen empresas diferentes, desarrollan y administran sus negocios bajo criterios y metas diferentes, muchas de ella tienen un enfoque más social que económico. Las mujeres cuentan con negocios más pequeños, se concentran en el sector servicios, su prioridad es conciliar trabajo y familia, y toman decisiones a partir de criterios diferentes; esto debido a que los negocios liderados por hombres se enfocan principalmente en aspectos financieros y económicos y realizan evaluaciones de inversiones y rentabilidad. A diferencia de las empresarias que priorizan la calidad de vida sobre la rentabilidad, presentan avances progresivos, con inversiones modestas, pues no cuentan con un capital importante, y utilizan sus propios recursos: conocimientos, gustos, formación educativa, inquietudes y experiencias de vida, buscando integrar su vida personal y familiar con su negocio o profesión, dándole mayor énfasis al equilibrio entre trabajo y familia; como consecuencia, las empresas de mujeres son más pequeñas y menos productivas y rentables (Camarena Adame \& Saavedra García, 2016; Valdivia, 2014). 
El escaso capital financiero con el que cuentan las empresarias al momento de iniciar sus empresas, tiene origen en la inequidad salarial que prevalece estableciendo una brecha que perjudica a las mujeres al recibir menos salario por el mismo trabajo, que un hombre, y se da con más fuerza en las sociedades donde existe mayor segregación por género, donde esta situación devalúa los salarios de todas las mujeres en todos los sectores económicos en los que trabajan (CEPAL, 2019; Tonoyan, Strohmeyer, \& Jennings, 2020; Tverdostup \& Paas, 2017).

En el sector servicios, donde se encuentran principalmente las empresas dirigidas por mujeres, la tecnología y el valor agregado son factores determinantes para el éxito de las empresas, a su vez, estos dependen de la educación, capacitación y capital; en estos rubros las PYMES de mujeres generalmente son carentes por lo que encuentran serias limitaciones para promover su desarrollo (Berger \& Kuckertz, 2016; SELA, 2010).

Guzmán y Rodríguez (2008) encontraron, que las empresas dirigidas por mujeres, son de tamaño reducido, generalmente operan en el sector servicios y cuentan con una tasa menor de supervivencia empresarial y son de una única propietaria. Por su parte, García Solarte, González Campo, y Murillo Vargas (2017) refieren que las PYME dirigidas por mujeres a diferencia de las dirigidas por hombres son pocas en los sectores de manufactura y alta tecnología, sin embargo las que operan en este sector son de mayor tamaño en cuanto al número de empleados; tienen menor participación femenina dentro de sus empleados, pero mayor participación dentro de las juntas directivas; no pertenecen ampliamente a organizaciones de carácter familiar; tienen mayores socios o dueños hombres y su nivel de formación mayoritariamente es en estudios básicos.

Inmujeres (2005), en un sondeo realizado en México señala como principales hallazgos que la mayoría de las empresas dirigidas por mujeres, operan en el sector servicios (41\%), seguidas por las del sector comercio (27\%), quedando en menor proporción las que operan en la industria (16\%), siendo esto coincidente con CEPAL (2019) que refiere acerca de la incipiente participación de las mujeres en la industria; en lo que se refiere al tamaño la mayoría son microempresas (55\%), enseguida aparecen las empresas pequeñas (39\%) y en mucho menor proporción la empresa mediana (6\%); el 38\% de las empresas tienen una antigüedad menor a 5 años, mientras que las que se encuentran entre 5 y 10 años representan el 30\% y las que tienen más de 10 años alcanzan el 32\%; las empresas de mayor antigüedad, son las que mostraron mayores ventas. En esta misma investigación se encontró que cuatro de cada cinco empresas fueron fundadas por las empresarias, por lo que la herencia familiar no fue determinante para emprender en este grupo de mujeres.

Por su parte Santhi (2017) en un estudio de empresas de mujeres en India encontró que el 87\% son pequeñas empresas, en cuanto al sector en el que operan, casi 18 de cada 100 refieren que sus negocios se dedicaban a los servicios profesionales como académicos, TI, etc., 27 mujeres tenían negocios de prendas de vestir, 44 estaban dedicadas a la industria de alimentos y bebidas y solo 11 a la industria manufacturera. Más de la mitad de las empresarias también manifestaron que iniciaron sus empresas con ahorros personales.

Así también, Value for Women (2018) refiere que en México las empresas dirigidas por mujeres son principalmente de tamaño micro, una gran parte de estas se encuentran en la informalidad, tienen ingresos bajos, lo que limita su potencial de crecimiento. Así también, una gran parte están en el sector servicios (73\%). Sin embargo, a pesar de esto Kalnins y Williams (2014) encontraron que las empresas propiedad de mujeres siempre sobreviven a las empresas propiedad de hombres en muchas industrias y regiones del mundo.

Por su parte GEM (2019) da cuenta que, a nivel mundial, el 53.4\% de las mujeres empresarias se dedican al comercio mayorista / minorista en comparación con el $43.5 \%$ de los hombres. Las mujeres emprendedoras tienen más probabilidades de estar presentes en el gobierno / salud / educación y servicios sociales. Las mayores brechas de género se encuentran en la agricultura, la minería y la tecnología de la información y las comunicaciones (TIC), donde los hombres emprendedores tienen más del doble de probabilidades que las mujeres de operar. Es importante destacar que 16 países informaron que no hay mujeres en las TIC (se estudiaron 59 países).

\section{Metodología}

\subsection{Muestra y cuestionario}

Se ha planteado un estudio cuantitativo de carácter descriptivo y exploratorio. La muestra se obtuvo de propietarias de 272 empresas PYME de la Ciudad de México. Se tomaron como criterios que la propietaria o accionista mayoritaria fuera mujer y que tuvieran de 4 a 250 empleados, considerando que la microempresa es la que tiene hasta 10 empleados, la pequeña empresa de 11 a 50 empleados y la mediana de 51 a 250 empleados (Secretaria de Economía, 2009). Los datos se recolectaron entre los meses de julio a diciembre de 2016, aplicando personalmente el cuestionario a las 272 empresarias que voluntariamente accedieron a colaborar con la investigación en las 16 delegaciones de la Ciudad de México. 
Se aplicó un cuestionario directo estructurado, para recolectar datos acerca de las variables e indicadores, sobre aspectos de capacitación, competitividad, finanzas, organización y toma de decisiones, redes y percepción de la empresaria; para efectos de este artículo solo se presentan los datos de la empresa, de la empresaria y la variable capacitación, que se describen a continuación.

\subsection{Esquema de variables analizadas}

En la Table 1 se presenta el listado de variables utilizadas en este estudio exploratorio. Los detalles sobre su medición y categorización se recogen en el apartado 4 de resultados.

Table 1. Variables e Indicadores del Cuestionario

\begin{tabular}{|c|c|}
\hline Variables & Indicadores \\
\hline Características de la empresa & $\begin{array}{l}\text { Sector, tamaño, antigüedad, personalidad jurídica, formalidad, propiedad del } \\
\text { establecimiento, competitividad del sector. }\end{array}$ \\
\hline $\begin{array}{l}\text { Perfil y características de la } \\
\text { mujer empresaria }\end{array}$ & $\begin{array}{l}\text { Edad, nivel de estudios, área de estudios, experiencia laboral y empresarial, } \\
\text { nivel de estudios de los padres, ocupación de los padres, razones para } \\
\text { emprender, barreras para emprender, propietaria de negocio anterior. }\end{array}$ \\
\hline Capacitación & $\begin{array}{l}\text { Adquisición de conocimientos empresariales, cursos de capacitación, áreas } \\
\text { de capacitación, curso de utilidad para el negocio, instituciones de } \\
\text { capacitación, motivos para capacitarse, duración de los cursos, solicitud de } \\
\text { cursos, programa de capacitación, áreas en que se capacita, motivos para } \\
\text { capacitar. }\end{array}$ \\
\hline
\end{tabular}

\section{Resultados}

\subsection{Características de las empresas y perfil de las mujeres empresarias}

Según los datos analizados en nuestro estudio, el $49 \%$ de las empresas pertenecen al sector servicios, mientras que el $39 \%$ corresponden al sector comercio y sólo el $12 \%$, son del sector industria, siendo coincidente con la literatura previa que indica que las empresas dirigidas por mujeres se concentran principalmente en servicios y comercio (Banco Mundial, 2010; Santhi, 2017; Value for Women, 2018; Zabludovsky, 2001).

En cuanto al tamaño, el 92\% corresponde a empresas de tamaño micro, el 7\% son empresas pequeñas y menos del $1 \%$ corresponden a empresas medianas, corroborando la literatura revisada donde se señala que las empresas dirigidas por mujeres son principalmente de tamaño micro (Espino, 2005; García Solarte et al., 2017; Guzmán \& Rodríguez, 2008; OIT, 2008; Santhi, 2017; Value for Women, 2018). El 80\% de las empresas son personas físicas o naturales, es decir carecen de personalidad jurídica. El 80\% de las empresas son formales mientras que el $20 \%$ son informales, a diferencia de lo que señalan la OIT (2008) y Value for Women (2018), acerca de que las empresas dirigidas por mujeres, principalmente se encuentran en la informalidad. Sólo el $36 \%$ de los establecimientos son propios, en tanto que el $60 \%$ son rentados y el $4 \%$ son prestados, a este respecto OIT (2008) ha señalado que esto se debe a que las empresarias tienen dificultades para conseguir instalaciones fijas, entre otras cosas debido a que carecen de derechos de propiedad y de sucesión. El $40 \%$ de las empresas tienen más de una década de existencia, mientras que el resto tienen menos antigüedad, destacándose las empresas en nacimiento (0-2 años) que son una tercera parte del total, coincidiendo esto con los hallazgos de Inmujeres (2005).

El $60 \%$ de las empresarias son mayores de 40 años, indicando este resultado que cuentan con alguna experiencia laboral antes de emprender, siendo coincidente este hallazgo con lo encontrado por Santhi (2017), Value for Women (2018) y Welsh et al. (2018), que señalan que a las mujeres maduras emprendedoras les puede resultar más fácil equilibrar los conflictos entre el trabajo y la familia, puesto que sus hijos son mayores y requieren menos atención; han ocupado principalmente puestos operativos; mientras que el $40 \%$ son menores de 40 años, mismas que emprenden con el fin de equilibrar el trabajo con las responsabilidades familiares, siendo coincidente los resultados de la edad con lo encontrado por Daeren (2000), Inmujeres (2005), Heller (2010) y De Vita et al. (2014).

En cuanto a los estudios realizados predomina el nivel de licenciatura (36\%) al igual que lo encontrado por Inmujeres (2005), Santhi (2017) y Value for Women (2018), en el área empresarial (46\%). Por otra parte, el $52 \%$ están casadas o tienen pareja, mientras el resto son solteras (35\%), y en menor proporción están divorciadas o viudas, resultado similar a lo encontrado por Inmujeres (2005) y De Vita et al. (2014), no así con lo encontrado por Santhi (2017) quién señala que el 75\% de las empresarias son casadas. En cuanto al nivel de estudios de los padres, en el padre predomina el nivel primaria (30\%), mientras que en la padre predomina también el nivel primaria (40\%), pero en una proporción superior; sólo el 15\% de las madres alcanzaron el nivel licenciatura, mientras que el $24 \%$ de los padres alcanzaron también este nivel, mostrando con esto que los padres tuvieron más oportunidades para desarrollarse en el ámbito profesional. 
Sólo una tercera parte de las empresarias manifestaron haber sido propietarias de un negocio anterior, contrario a los hallazgos de Inmujeres (2005) donde el 70\% de las entrevistadas manifestaron contar con algún antecedente empresarial, lo cual resulta relevante pues Tonoyan et al. (2020) refieren que las mujeres se inclinan por negocios en los cuales tienen experiencia puesto que les permite aprovechar los conocimientos adquiridos.

\subsection{La capacitación en las empresas dirigidas por mujeres}

Tal y como se muestra en la Figure 1, entre los empleados -tanto en hombres como en mujeres- de las empresas encuestadas predominan los estudios de secundaria, seguido de los estudios de bachillerato, lo que muestra que los empleados no cuentan con preparación especializada para realizar su trabajo, sino nada más con conocimientos generales coincidiendo con los hallazgos del Banco Mundial (2010), en el que señalan que las empresas de mujeres tienen un capital humano con menor preparación profesional. Así también, Saavedra García y Camarena Adame (2015) refieren que las mujeres trabajan un capital humano de menor calidad que los hombres quienes prefieren personal más capacitado y experto. De ahí entonces, la importancia de impulsar la capacitación en estas empresas, con el fin de enriquecer el capital humano.

Figure 1. Nivel de estudios de los empleados

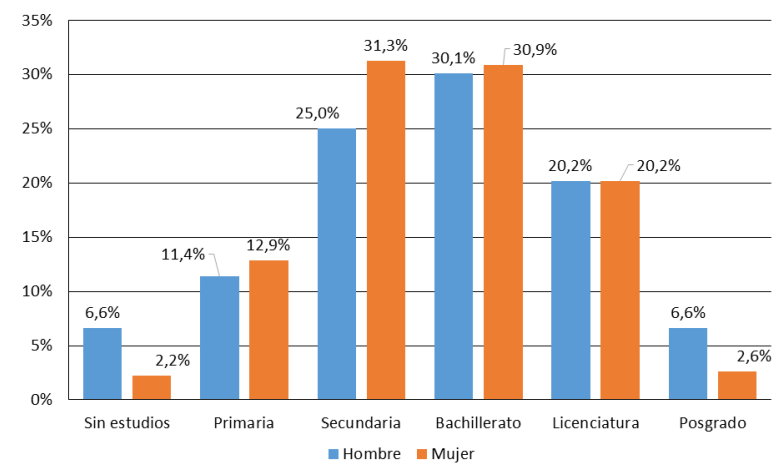

Fuente: Elaboración propia

El 73.50\% de las empresarias manifiestan que la escuela le permitió adquirir conocimientos empresariales, mientras que $66.20 \%$ manifestaron que también obtuvieron conocimientos de la familia, coincidiendo con los estudios de Inmujeres (2005), Inmujeres (2009) y Lopez Sullivan (2013). Sin embargo, aun cuando el 89\% consideran importantes los cursos de capacitación empresarial sólo un poco más de la mitad los ha tomado (ver Table 2), esto se debería a la falta de tiempo (Value for Women, 2018), por un lado, y por el otro a que las mujeres tienen menos probabilidades de conseguir capacitación y servicios de desarrollo empresarial, como lo ha señalado el estudio realizado por Banco Mundial (2010) o también porque encuentran los cursos poco útiles al ser poco aplicables a su realidad especifica (Value for Women, 2018). Esto resulta preocupante puesto que SELA (2010), Santhi (2017) y Jena (2018) señalan que las empresarias se enfrentan a la falta de conocimiento sobre como dirigir una empresa, lo que no les da seguridad al momento de tomar decisiones, por lo que la capacitación estaría equilibrando esta falla.

Table 2. Conocimientos empresariales y cursos

\begin{tabular}{|l|c|c|}
\hline \multicolumn{1}{|c|}{ Ítem } & Sí & No \\
\hline Conocimiento de la escuela & $73.5 \%$ & $26.5 \%$ \\
\hline Conocimiento de la familia & $66.2 \%$ & $33.8 \%$ \\
\hline Es importante tomar cursos de capacitación empresarial & $89.0 \%$ & $11.0 \%$ \\
\hline Ha tomado cursos de capacitación empresarial & $54.8 \%$ & $45.2 \%$ \\
\hline Fuente: Elaboración propia & &
\end{tabular}

Las áreas en las que principalmente han tomado los cursos de capacitación son administración y contabilidad, así como áreas relacionadas con la comercialización de los bienes y servicios que ofrecen, siendo coincidente con lo que refieren Bauer, Finnegan, y Haspels (2004), Santhi (2017), CEPAL (2019), en lo que se refiere a la formación en administración. Asimismo, manifiestan que estos cursos han sido de utilidad para el negocio (Ver Table 3). 
Table 3. Áreas de capacitación

\begin{tabular}{|l|r|r|}
\hline & \multicolumn{1}{|c|}{ Sí } & No \\
\hline Administración y contabilidad & $54.4 \%$ & $45.6 \%$ \\
\hline Comercialización, mercadotecnia, promoción, publicidad y ventas & $43.6 \%$ & $56.4 \%$ \\
\hline Aspectos de organización & $30.9 \%$ & $69.1 \%$ \\
\hline Computación & $29.5 \%$ & $70.5 \%$ \\
\hline Desarrollo humano & $28.2 \%$ & $71.8 \%$ \\
\hline Recursos Humanos & $26.8 \%$ & $73.2 \%$ \\
\hline Seguridad e higiene & $26.2 \%$ & $73.8 \%$ \\
\hline Aspectos fiscales y contables & $23.5 \%$ & $76.5 \%$ \\
\hline Producción & $22.1 \%$ & $77.9 \%$ \\
\hline Finanzas & $22.1 \%$ & $77.9 \%$ \\
\hline Idiomas & $16.8 \%$ & $83.2 \%$ \\
\hline Contaminación y medio ambiente & $12.8 \%$ & $87.2 \%$ \\
\hline Manejo, mantenimiento y reparación de equipos y maquinaria & $9.4 \%$ & $90.6 \%$ \\
\hline Comercio exterior & $8.0 \%$ & $92.0 \%$ \\
\hline
\end{tabular}

Fuente: Elaboración propia

Las empresarias se capacitaron principalmente en instituciones privadas (26.8\%) y gubernamentales (26.8\%). Este hallazgo permite saber que las empresarias estarían dispuestas a pagar por la capacitación, dado que consideran importante contar con esta, por otra parte, las asociaciones empresariales y las escuelas públicas no les resultan atractivas para capacitarse (Ver Table 4). Sin embargo, Cho et al. (2019), encontraron que las empresarias prefieren los talleres gratuitos, seminarios y clases interactivas, a través de sitios web, blocks y videos; así también, sugieren que el contacto con otros empresarios y mentores les brindaría un buen entrenamiento para gestionar sus empresas. Lo anterior implica que conocer historias de éxito de empresarios es una vía de aprendizaje importante para las mujeres. Por su parte, Varadarajan Sowmya, Majumdar, y Gallant (2010) encontraron que las mujeres prefieren ser orientadas por medio de programas empresariales especializados.

Table 4. Instituciones de capacitación

\begin{tabular}{|l|r|}
\hline & Porcentaje \\
\hline Escuela privada & $26.8 \%$ \\
\hline Institución gubernamental & $26.8 \%$ \\
\hline Asociación empresarial & $16.8 \%$ \\
\hline Escuela pública & $12.8 \%$ \\
\hline Institución financiera & $4.0 \%$ \\
\hline Otra & $12.8 \%$ \\
\hline Total & $\mathbf{1 0 0 . 0} \%$ \\
\hline
\end{tabular}

Fuente: Elaboración propia

El principal motivo por el cual la empresaria decidió capacitarse es para resolver los problemas que se presentan en la empresa y para mejorar su manejo, así también se encuentran interesadas en adquirir y ampliar sus conocimientos (Ver Table 5), coincidiendo este hallazgo con lo encontrado por el Banco Mundial (2010), donde las empresarias señalan que la capacitación es muy importante y resulta ser de gran beneficio para impulsar sus empresas. Esta motivación de las empresarias resulta relevante pues la capacitación se encuentra relacionada con la competitividad y la productividad (Camarena Adame \& Saavedra García, 2016; Sapién Aguilar et al., 2014) y le brinda seguridad a la empresaria (Espino, 2018).

Table 5. Motivos para capacitarse

\begin{tabular}{|l|r|}
\hline & Porcentaje \\
\hline Resolver algún problema o mejorar el manejo de la empresa & $37.6 \%$ \\
\hline Adquirir conocimientos sobre el tema & $30.2 \%$ \\
\hline Ampliar sus conocimientos sobre el tema & $24.2 \%$ \\
\hline Porque era un requisito para poder recibir un apoyo & $3.3 \%$ \\
\hline Otro & $4.7 \%$ \\
\hline Total & $\mathbf{1 0 0 . 0} \%$ \\
\hline
\end{tabular}

Fuente: Elaboración propia

Las empresarias tomaron en promedio 45 horas de capacitación al año y manifestaron que implementaron en su negocio lo aprendido en el curso el 98\% de las mismas, superando este porcentaje lo encontrado por Intersocial (2011) en donde el 64\% de las mujeres aplicaron los conocimientos obtenidos en la capacitación. Sin embargo, se considera que es insuficiente el tiempo que la empresaria le dedica a la capacitación dado que no representa ni siquiera una hora a la semana (Monge González \& Rodríguez Álvarez, 2014), lo anterior, se debería entre otros aspectos a la responsabilidad familiar que aún sigue recayendo sobre ella (Value for Women, 2018). Esta es una situación importante y que se debe atender para que la empresaria reciba más capacitación dado que esta le permitirá el desarrollo de habilidades gerenciales clave, que le permitirá planear 
y organizar el trabajo, brindándole la capacidad de motivar y entrenar a otros, reduciendo el ausentismo, la rotación y elevando la motivación de su personal, lo cual le traerá resultados empresariales positivos (Panagiotakopoulos, 2020; Tverdostup \& Paas, 2017).

Las empresarias desean seguir tomando cursos de administración y contabilidad, así como de comercialización, aunado a esta más de una cuarta parte también mostraron interés en temas financieros, fiscales y de recursos humanos (Ver Table 6), coincidiendo con lo señalado por Santhi (2017), dado que carecen de este tipo de conocimientos (Cho et al., 2019); este resultado es relevante pues de acuerdo con Inmujeres (2009) una estrategia de capacitación integral es necesaria para que las mujeres asimilen mejor los conocimientos e implementen iniciativas de negocio para mejorar su gestión empresarial.

Table 6. Cursos deseados

\begin{tabular}{|l|r|}
\hline Administración y contabilidad & Porcentaje \\
\hline Comercialización, mercadotecnia, promoción, publicidad y ventas & $40.8 \%$ \\
\hline Finanzas & $34.9 \%$ \\
\hline Aspectos fiscales y contables & $27.9 \%$ \\
\hline Recursos Humanos & $26.1 \%$ \\
\hline Computación & $25.7 \%$ \\
\hline Seguridad e higiene & $23.2 \%$ \\
\hline Desarrollo humano & $20.6 \%$ \\
\hline Producción & $20.2 \%$ \\
\hline Aspectos de organización & $21.0 \%$ \\
\hline Idiomas & $19.9 \%$ \\
\hline Comercio exterior & $19.5 \%$ \\
\hline Contaminación y medio ambiente & $14.7 \%$ \\
\hline Manejo, mantenimiento y reparación de equipos y maquinaria & $10.7 \%$ \\
\hline Fuente: Elaborán propia & $9.9 \%$ \\
\hline
\end{tabular}

Fuente: Elaboración propia

Sólo el 32\% de las empresas lideradas por mujeres cuentan con programas de capacitación, lo cual es opuesto a lo encontrado por Sapién Aguilar et al. (2014) donde el 76\% de las empresas medianas cuentan con programas de capacitación y con lo encontrado por Quevedo Batista, Ruiz Lopez, Antonio Vidaña, y Muñoz Palacios (2017) donde el 49\% de las PYME estudiadas cuentan con un programa de capacitación; las empresas analizadas en esta investigación capacitan en las siguientes áreas (Ver Table 7):

Table 7. Áreas en las que capacita

\begin{tabular}{|l|r|}
\hline Producción/operaciones & Porcentaje \\
\hline Mercadotecnia & $66.7 \%$ \\
\hline Recursos humanos & $12.6 \%$ \\
\hline Finanzas (contable) & $5.7 \%$ \\
\hline Otra & $4.7 \%$ \\
\hline Total & $10.3 \%$ \\
\hline Fuente: Elaboración propia & $\mathbf{1 0 0 . 0} \%$ \\
\hline
\end{tabular}

Como se puede apreciar en la Table 7, las áreas prioritarias de capacitación son las operativas, siendo coincidente con los hallazgos de Sapién Aguilar et al. (2014), esto dado a que la empresaria está preocupada por el día a día en la empresa, es decir su visión es de corto plazo. Por otra parte, de acuerdo con Inmujeres (2009) y Santhi (2017) las mujeres señalan que la falta de capacitación técnica se encuentra entre los principales problemas que enfrentan, por lo que capacitar en esta área les puede ayudar a resolver éstos. Este es un aspecto que se debería reforzar, pues estudios recientes señalan que mejorar las habilidades de todo tipo de trabajadores, incluidos los gerentes, es fundamental para mejorar el desempeño de la empresa, sobre todo para las de tamaño pequeño (Panagiotakopoulos, 2020).

Las empresarias manifestaron que el principal motivo para capacitar es para mejorar los resultados y aumentar las ganancias (ver Table 8), coincidiendo con los hallazgos de Valdivia (2014) e Intersocial (2011) quienes comprobaron que la asistencia técnica acompañada de capacitación en primer lugar, trae como resultado un crecimiento rápido en el corto plazo; en segundo lugar, queda el adiestramiento que tiene relación directa con el incremento de la productividad y en tercero la inducción a la empresa que es de utilidad para crear sentido de pertenencia a la empresa. Otros autores refieren la importancia de la capacitación, dada su relación con la productividad y competitividad, así como, la disminución de riesgos de trabajo (Sapién Aguilar et al., 2014); por otra parte, Espino (2018) destaca la mejora en la autoestima e interacción social del personal capacitado. 
Table 8. Motivos para capacitar

\begin{tabular}{|l|r|}
\hline & Porcentaje \\
\hline mejorar los resultados/ aumentar las ganancias & $40.2 \%$ \\
\hline Adiestramiento & $26.4 \%$ \\
\hline Inducción & $23.0 \%$ \\
\hline Para obtener una promoción & $2.3 \%$ \\
\hline Otra & $8.1 \%$ \\
\hline Total & $\mathbf{1 0 0 . 0} \%$ \\
\hline
\end{tabular}

Fuente: Elaboración propia

\subsection{Relación entre capacitación, perfil de la mujer empresaria y características de la empresa}

La tabla de contingencia y la chi-cuadrado han servido como instrumento de análisis de la relación entre la capacitación (medida mediante la variable dicotómica “cuenta con programa de capacitación” y de respuesta Sí/No) y las características de la empresaria.

Table 9. Relación entre las características de la empresaria y la capacitación

\begin{tabular}{|c|c|c|c|}
\hline & \multicolumn{2}{|c|}{ Capacitación } & \multirow[t]{2}{*}{ Valor de p } \\
\hline & Sí & No & \\
\hline \multicolumn{4}{|l|}{ Edad (\%) } \\
\hline 18-30 años & $38,7 \%$ & $61,3 \%$ & \multirow[t]{4}{*}{0,462} \\
\hline 31-40 años & $23,4 \%$ & $76,6 \%$ & \\
\hline 41-60 años & $24,6 \%$ & $75,4 \%$ & \\
\hline 60 años a más & $30,6 \%$ & $69,4 \%$ & \\
\hline \multicolumn{4}{|l|}{ Nivel de estudios } \\
\hline Primaria & $4,3 \%$ & $95,7 \%$ & \multirow[t]{5}{*}{0,002} \\
\hline Secundaria & $19,2 \%$ & $80,8 \%$ & \\
\hline Bachillerato & $23,1 \%$ & $76,9 \%$ & \\
\hline Licenciatura & $38,8 \%$ & $61,2 \%$ & \\
\hline Posgrado & $50,0 \%$ & $50,0 \%$ & \\
\hline \multicolumn{4}{|l|}{ Área de estudios } \\
\hline Empresarial & $42,1 \%$ & $57,9 \%$ & \multirow[t]{5}{*}{0,005} \\
\hline Físico, matemáticas e Ingenierías & $22,2 \%$ & $77,8 \%$ & \\
\hline Ciencias biológicas y de la salud & $27,8 \%$ & $72,2 \%$ & \\
\hline Humanidades y artes & $33,3 \%$ & $66,7 \%$ & \\
\hline Ciencias Sociales & $41,2 \%$ & $58,8 \%$ & \\
\hline \multicolumn{4}{|l|}{ Nivel de estudios de la madre } \\
\hline Primaria & $23,8 \%$ & $76,2 \%$ & \multirow[t]{5}{*}{0,002} \\
\hline Secundaria & $27,5 \%$ & $72,5 \%$ & \\
\hline Bachillerato & $31,7 \%$ & $68,3 \%$ & \\
\hline Licenciatura & $37,2 \%$ & $62,8 \%$ & \\
\hline Posgrado & $100,0 \%$ & $0,0 \%$ & \\
\hline \multicolumn{4}{|l|}{ Nivel de estudios del padre } \\
\hline Primaria & $22,6 \%$ & $77,4 \%$ & \multirow[t]{5}{*}{0,347} \\
\hline Secundaria & $22,0 \%$ & $78,0 \%$ & \\
\hline Bachillerato & $40,0 \%$ & $60,0 \%$ & \\
\hline Licenciatura & $31,8 \%$ & $68,2 \%$ & \\
\hline Posgrado & $50,0 \%$ & $50,0 \%$ & \\
\hline \multicolumn{4}{|l|}{ Puestos en los que ha trabajado } \\
\hline No ha trabajado & $17,9 \%$ & $82,1 \%$ & \multirow[t]{4}{*}{0,136} \\
\hline Operativo & $42,1 \%$ & $57,9 \%$ & \\
\hline Mandos medios & $32,9 \%$ & $67,1 \%$ & \\
\hline Directivo & $29,7 \%$ & $70,3 \%$ & \\
\hline \multicolumn{4}{|l|}{ Tipo de experiencia laboral } \\
\hline No tiene experiencia & $16,2 \%$ & $83,8 \%$ & \multirow[t]{3}{*}{0,035} \\
\hline En otro giro & $30,3 \%$ & $69,7 \%$ & \\
\hline En el mismo giro & $50,7 \%$ & $49,3 \%$ & \\
\hline \multicolumn{4}{|l|}{ Propietario de un negocio anterior } \\
\hline $\mathrm{Si}$ & $46,9 \%$ & $53,1 \%$ & \multirow[t]{2}{*}{0,028} \\
\hline No & $23,9 \%$ & $76,1 \%$ & \\
\hline
\end{tabular}

Fuente: Elaboración propia

Los resultados se presentan en la Table 9. El nivel de estudios tiene relación con la capacitación, analizando la tabla de contingencia se puede saber que a mayor nivel de estudios resulta más elevado el porcentaje empresas que cuentan con programas de capacitación, pues de las empresarias que implementan programas de capacitación son el 23\% de las que cuentan con nivel de Bachillerato, el 39\% de las que tienen estudios de 
licenciatura y 50\% de las que alcanzaron el nivel de maestría. Demostrando así, que cuando más elevado es el nivel de estudios de la empresaria, mayor probabilidad existe de que implemente en su empresa programas de capacitación.

El área de estudios tiene relación con la capacitación, así pues, con base en los resultados de la tabla de contingencia se puede afirmar que las empresarias con estudios en áreas empresariales y ciencias sociales, muestran una mayor tendencia a implementar programas de capacitación, pues los estudios antecedentes señalan que existe relación entre poseer una carrera empresarial, la motivación por el logro y el buen desempeño empresarial (Kundu \& Rani, 2016). Estos programas, son implementados sólo por un $22 \%$ de las que cuentan con estudios en el área de física, matemáticas e ingenierías, $28 \%$ ciencias biológicas y de la salud, $33 \%$ artes y humanidades, $41 \%$ Ciencias sociales y humanidades y $42 \%$ áreas empresariales. Lo que estaría indicando que las empresarias que pertenecen a estas dos últimas áreas se encuentran más sensibilizadas acerca de la importancia de contar con un capital humano de mayor valor.

El nivel de estudios de la madre tiene relación con la capacitación, en este caso analizando la tabla de contingencia se puede notar que las empresarias con madres que han obtenido niveles de estudios más elevados, mayormente cuentan con programas de capacitación en sus empresas. Esto se debería, a que el nivel de estudios de la madre tiene relación positiva directa con el nivel de estudios de la empresaria (61\%, calculado con el coeficiente de correlación de Spearman).

El tipo de experiencia laboral tiene relación con la capacitación, así pues, la experiencia en el mismo giro, destaca en las empresarias que cuentan con programas de capacitación, dado que el 51\% de estas manifestaron contar con estos programas, mientras que las que tenían experiencia en otras áreas solo fueron el $30 \%$.

Las empresarias que tuvieron un negocio anterior, son las que cuentan en una proporción más elevada con programas de capacitación en sus empresas, lo que implica que la mayor experiencia empresarial les permite visualizar la importancia de contar con este programa en sus empresas.

Se ha utilizado de nuevo la tabla de contingencia y la chi cuadrado para establecer la relación entre la capacitación y las características de la empresa. Los resultados, mostrados en la Table 10, indican que la personalidad jurídica tiene relación con la capacitación. Cuando la empresa es persona moral o sociedad mercantil, mayor es la tendencia a contar con programas de capacitación (30\% si son personas físicas y $70 \%$ si son personas morales). A partir de este hallazgo se puede inferir que las empresas con la figura de persona moral tienden a contar con una organización más profesional.

Table 10. Relación entre las características de la empresa y la capacitación

\begin{tabular}{|c|c|c|c|}
\hline & \multicolumn{2}{|c|}{ Capacitación } & \multirow[t]{2}{*}{ Valor de p } \\
\hline & Sí & No & \\
\hline \multicolumn{4}{|l|}{ Sector } \\
\hline Industria & $42,4 \%$ & $57,6 \%$ & \multirow[t]{3}{*}{0,080} \\
\hline Comercio & $24,5 \%$ & $75,5 \%$ & \\
\hline Servicio & $35,3 \%$ & $64,7 \%$ & \\
\hline \multicolumn{4}{|l|}{ Antigüedad } \\
\hline 0-2 años & $33,3 \%$ & $66,7 \%$ & \multirow[t]{5}{*}{0,226} \\
\hline 3- 4 años & $46,9 \%$ & $53,1 \%$ & \\
\hline 5-8 años & $21,4 \%$ & $78,6 \%$ & \\
\hline 0-12 años & $22,7 \%$ & $77,3 \%$ & \\
\hline Más de 12 años & $31,2 \%$ & $68,8 \%$ & \\
\hline \multicolumn{4}{|c|}{ Personalidad Jurídica } \\
\hline Persona Física & $30,1 \%$ & $69,9 \%$ & \multirow[t]{2}{*}{0,011} \\
\hline Persona Moral & $60,9 \%$ & $39,1 \%$ & \\
\hline \multicolumn{4}{|l|}{ Formal/Informal } \\
\hline Formal & $35,7 \%$ & $64,3 \%$ & \multirow[t]{2}{*}{0,168} \\
\hline Informal & $32,2 \%$ & $67,8 \%$ & \\
\hline \multicolumn{4}{|c|}{ Establecimiento Propio/Rentado } \\
\hline Propio & $30,6 \%$ & $69,4 \%$ & \multirow[t]{2}{*}{0,265} \\
\hline Rentado & $34,1 \%$ & $65,9 \%$ & \\
\hline \multicolumn{4}{|l|}{ Tamaño } \\
\hline Micro & $29,1 \%$ & $70,9 \%$ & \multirow[t]{3}{*}{0,001} \\
\hline Pequeña & $65,0 \%$ & $35,0 \%$ & \\
\hline Mediana & $100,0 \%$ & $0,0 \%$ & \\
\hline \multicolumn{4}{|c|}{ Competitividad del sector } \\
\hline Bajo crecimiento & $16,7 \%$ & $83,3 \%$ & \multirow[t]{3}{*}{0,010} \\
\hline Medio crecimiento & $29,8 \%$ & $70,2 \%$ & \\
\hline Alto crecimiento & $44,0 \%$ & $56,0 \%$ & \\
\hline
\end{tabular}


El tamaño de la empresa tiene relación con la capacitación, el resultado de la tabla de contingencia permite saber que el 29\% de las empresas de tamaño micro, el 65\% de las de tamaño pequeño y el $100 \%$ de las empresas medianas dirigidas por mujeres, cuentan con programas de capacitación. Lo cual determina que cuando más grande es la empresa mayor posibilidad existe que cuente con programa de capacitación, lo cual tendría relación con la mayor cantidad de recursos que posee en función de su dimensión.

La competitividad del sector (medida como el crecimiento del sector) tiene relación con la capacitación, analizando la tabla de contingencia se puede ver que de las empresas que cuentan con programa de capacitación, $16 \%$ pertenecen a un sector de bajo crecimiento, mientras que el $30 \%$ se encuentran en un sector de crecimiento medio y $44 \%$ son de un sector de alto crecimiento. Este hallazgo estaría indicando que la capacitación impulsa la competitividad, coincidiendo con los estudios realizados por Valdivia (2014) en el que las empresarias que se capacitaron obtuvieron en dos años un incremento en ventas de 15\%; y con lo encontrado por Intersocial (2011) donde el 64\% de las empresarias mejoraron sus ingresos luego de implementar los conocimientos obtenidos en la capacitación, así como con lo señalado por Sapién Aguilar et al. (2014) y Camarena Adame y Saavedra García (2016), acerca de la relación positiva existente entre la competitividad y la capacitación.

De acuerdo con los resultados anteriores, se concluye que contar con programa de capacitación está relacionado con el nivel de estudios de la empresaria y de la madre, el área de estudios, la experiencia laboral y el haber sido propietaria de un negocio anterior. Asimismo, se concluye que contar con programa de capacitación se encuentra relacionado con el tamaño, la personalidad jurídica y la competitividad del sector.

\section{Conclusiones finales}

La importancia de la mujer empresaria radica en su aporte no solo al desarrollo económico sino también al aporte que es capaz de realizar para mejorar la calidad de vida de su familia y con ello de la sociedad en la que está inmersa. Un factor que estaría limitando su desempeño es la falta de capacitación, debido a los sesgos que existen en referencia al género, que la orillan a seguir estudios típicos a su naturaleza de mujer y que no son más que una extensión de sus labores como ama de casa, es decir, servicios y cuidados; alejándola así de los conocimientos que podrían empoderarla en el ámbito empresarial, como por ejemplo el tecnológico.

Los resultados empíricos de esta investigación dejan saber que las mujeres empresarias de la Ciudad de México son conscientes de la importancia de la capacitación, sin embargo, al encontrarse en sectores de baja competitividad y crecimiento, no han implementado programas de capacitación en sus empresas, y los cursos que han tomado han sido básicamente para resolver problemas operativos y de corto plazo, lo que deja ver la falta de visión de la mujer empresaria.

Uno de los hallazgos a destacar es la relación que existe entre el nivel de estudios de la empresaria y de su madre con la capacitación, lo que es consistente puesto que una madre con un nivel de estudios superior impulsa a sus hijos para alcanzar mejores niveles que ella. A la vez una empresaria con estudios profesionales es más consciente de la importancia de la capacitación en la empresa, lo que es un indicador de una visión a largo plazo, con lo que se deja ver que a empresaria estaría buscando estabilidad y permanencia.

Las empresas constituidas con personalidad jurídica, de mayor tamaño y en sectores de alto crecimiento, son las que principalmente cuentan con programa de capacitación, destacando esto que se necesita tener una administración, profesionalizada y recursos suficientes para llevar a cabo estos programas.

Los resultados de este trabajo, permiten destacar la importancia de la capacitación para un mejor desempeño empresarial, pero también hacen un llamado de atención a las autoridades que operan los entes de fomento empresarial con el fin de que entiendan la necesidad de enfocar los apoyos dirigidos específicamente a la mujer empresaria.

\section{Acknowledgements}

Researh funded by: Dirección General de Asuntos del Personal Académico, Universidad Nacional Autónoma de México (Funder Identifier: http://dx.doi.org/10.13039/501100006087). Award: PE300415

\section{FootNotes}

${ }^{1} \mathrm{El}$ "techo de cristal” son los obstáculos que impiden que una mujer alcance puestos de alto nivel en las organizaciones (Camarena Adame \& Saavedra García, 2018), y se encuentra conformado por factores invisibles, como los estereotipos de género y prejuicios, políticas empresariales desfavorables y falta de experiencia para acceder a puestos gerenciales, que inciden en esta situación (CEPAL, 2019). 


\section{References}

ANUIES (2018). Visión y acción 2030 Propuesta de la ANUIES para renovar la educación superior en México. México D.F., México: Asociación Nacional de Universidadese Instituciones de Educación Superior. Retrieved from http://www.anuies.mx/media/docs/avisos/pdf/VISION_Y_ACCION_2030.pdf

Abramo, L., Valenzuela, M. E., \& Pollack, M. (2000). Equidad de género en el mundo del trabajo en América Latina. Avances y desafíos cinco años después de Beijing. Retrieved from http://white.lim.ilo.org/spanish/260ameri/publ/docutrab/dt-130/texto_completo.pdf

Attorresi, R. L. (2009). Capacitación en PyMes. Herramienta de gestión para generar conocimiento específico, lograr el desarrollo sostenido y acompañar el crecimiento. Rosario, Pellegrini: Universidad Abierta Interamericana

Banco Mundial (2010). Mujeres Empresarias: Barreras y oportunidades en el Sector Privado Formal en América Latina y el Caribe. Washington, D.C.: Banco Mundial

Bauer, S., Finnegan, G., \& Haspels, N. (2004). Get Ahead for Women in Enterprise Training Package and Resource Kit. Switzerland: International Labour Organization

Berger, E. S. C., \& Kuckertz, A. (2016). Female entrepreneurship in startup ecosystems worldwide. Journal of Business Research, 69(11), 5163-5168. https://doi.org/10.1016/j.jbusres.2016.04.098

Bloom, N., Eifert, B., Mahajan, A., McKenzie, D., \& Roberts, J. (2011). Does Management Matter? Evidence from India. Cambridge, MA: National Bureau of Economic Research. https://doi.org/10.3386/w16658

Brixiová, Z., Kangoye, T., \& Said, M. (2020). Training, human capital, and gender gaps in entrepreneurial performance. Economic Modelling, 85, 367-380. https://doi.org/10.1016/j.econmod.2019.11.006

Buquet, A., \& Moreno, H. (2011). Trayectorias de mujeres. Educación técnico profesional y trabajo en México. Santiago de Chile: Naciones Unidas, CEPAL. Retrieved from https://repositorio.cepal.org/handle/11362/41567

CEPAL (2019). La autonomía de las mujeres en escenarios económicos cambiantes. Santiago de Chile: Comisión Económica para América Latina y el Caribe

CEPAL (2019). Panorama Social de América Latina. Santiago de Chile: ONU, CEPAL

Camarena Adame, M. E., \& Saavedra García, M. L. (2016). The Relationship between Human Capital and the Competitiveness of SMEs in Mexico City, Mexico. British Journal of Economics, Management \& Trade, 14(1), 1-13. https://doi.org/10.9734/BJEMT/2016/26460

Camarena Adame, M. E., \& Saavedra García, M. L. (2016). Un estudio de las PYME lideradas por mujeres en Latinoamérica. Revista Universitaria Ruta, 18(1), 1-27. Retrieved from https://revistas.userena.cl/index.php/ruta/article/view/789

Camarena Adame, M. E., \& Saavedra García, M. L. (2018). El techo de Cristal en México. Revista de Estudios de Género, La Ventana, 5(47), 312-339. https://doi.org/10.32870/lv.v5i47.6680

Cho, E., Moon, Z. K., \& Bounkhong, T. (2019). A qualitative study on motivators and barriers affecting entrepreneurship among Latinas. Gender in Management: An International Journal, 34(4), 326-343. https://doi.org/10.1108/GM-07-2018-0096

Correia Santos, S. (2015). Emprendimiento femenino en Iberoamérica. España: RedEmprendia

Daeren, L. (2000). Mujeres empresarias en Latinoamérica: El difícil equilibrio entre dos mundos de trabajo. Paper presented at Primer Seminario Internacional de la MujerEmpresaria: SIME 2000, Santiago de Chile, 25 de agosto de 2000. Retrieved from

https://www.cepal.org/mujer/proyectos/gtz/publicaciones/word_doc/empresarias.pdf

De Vita, L., Mari, M., \& Poggesi, S. (2014). Women entrepreneurs in and from developing countries: Evidences from the literature. European Management Journal, 32(3), 451-460. https://doi.org/10.1016/j.emj.2013.07.009

Deka, R. J. (2018). A Study on the Importance of Skill Development: Women Entrepreneurs in India as a Catalyst to Women Empowerment.. Productivity, 58(4), 400-409

Elizundia Cisneros, M. E. (2015). Desempeño de nuevos negocios: perspectiva de género. Contaduría y Administración, 60(2), 468-485. https://doi.org/10.1016/S0186-1042(15)30010-3

Espino, A. (2005). Un marco de análisis para el fomento de las políticas de desarrollo productivo con enfoque de género (Serie Muje). Santiago de Chile: CEPAL

Espino, A. (2018). Capacitación laboral para la autonomía económica de las mujeres en situación de pobreza. Santiago de Chile: CEPAL

GEM (2019). Global Entrepreneurship Monitor 2018/2019 Women's Entrepreneurship Report (Vol. 8). London: Global Entrepreneurship Research Association, London Business School

García Solarte, M., González Campo, C. H., \& Murillo Vargas, G. (2017). Características de las pymes según la gerencia sea hombre o mujer: caso empírico de Cali. Revista EAN, 82.

https://doi.org/10.21158/01208160.n82.2017.1648 
Guzmán, J., \& Rodríguez, M. J. (2008). Comportamiento de las mujeres empresarias: una visión global. Revista de Economía Mundial, 18, 381-392

Heller, L. (2010). Mujeres emprendedoras en América Latina y el Caribe: realidades, obstáculos y desafíos (Vol. 93). Santiago de Chile: Naciones Unidas, CEPAL. Retrieved from http://repositorio.cepal.org/bitstream/handle/11362/5818/1/S0900676_es.pdf

Hernández, E. P. (2015). Empresarias y ejecutivas en México. Diagnósticos y desafíos. Espiral estudios sobre Estado y sociedad, 21(61), 261-267. https://doi.org/10.32870/eees.v21i61.271

Hillmert, S. (2015). Gender Segregation in Occupational Expectations and in the Labour Market: International Variation and the Role of Education and Training Systems. In: Gender Segregation in Vocational Education. Comparative Social Research (pp. 123-148). Bingley, UK: Emerald Group Publishing Limited. https://doi.org/10.1108/S0195-631020150000031005

IICA (1996). Liderazgo y género en el desarrollo rural sostenible. Managua: Instituto Interamericano de Cooperación para la Agricultura

INEGI (2018). Mujeres y hombres en México, 2018. México: Instituto Nacional de Estadística y Geografía (México). Retrieved from http://cedoc.inmujeres.gob.mx/documentos_download/MHM_2018.pdf

Inmujeres (2005). Las mujeres empresarias en México. México: Instituto Nacional de las mujeres. Retrieved from http://cedoc.inmujeres.gob.mx/documentos_download/100583.pdf

Inmujeres (2009). Empresas de mujeres = Empresas exitosas. México: Instituto Nacional de las mujeres

Intersocial (2011). Evaluación final y externa del proyecto: Mejora de la capacitación técnica de Mujeres de escasos recursos de cañete para la actividad económica Sostenible. Perú: Centro de asesoría para la intervención social, Intersocial, S.A.C.

Jena, R. A. (2018). Women Entrepreneurship : A synoptic view. ASCI Journal of Management, 47(1), 45-58. Retrieved from https://asci.org.in/journal/Journal-47(1)MARCHsplissue.pdf

Kalnins, A., \& Williams, M. (2014). When do female-owned businesses out-survive male-owned businesses? A disaggregated approach by industry and geography. Journal of Business Venturing, 29(6), 822-835. https://doi.org/10.1016/j.jbusvent.2013.12.001

Kray, L. J., \& Kennedy, J. A. (2017). Changing the Narrative: Women as Negotiators-and Leaders. California Management Review, 60(1), 70-87. https://doi.org/10.1177/0008125617727744

Kundu, S. C., \& Rani, S. (2016). Female aspirant human resources' entrepreneurial orientation. Management Research Review, 39(2), 235-263. https://doi.org/10.1108/MRR-11-2015-0267

Lopez Sullivan, E. J. (2013). Las mujeres en empresas, profesiones y ocupaciones no tradicionales: La experiencia de Puerto Rico. Paper presented at Global Conference on Business and Finance Proceedings, San José, Costa Rica. Retrieved from http://www.theibfr.com/ARCHIVE/ISSN-1941-9589-V8-N2-2013.pdf

Monge González, R., \& Rodríguez Álvarez, J. A. (2014). El impacto de los servicios financieros y la capacitación de las MIPYMES de Costa Rica. TEC Empresarial, 8(1), 19. https://doi.org/10.18845/te.v8i1.1722

OIT (2008). La estrategia de la OIT para promover la iniciativa empresarial de la mujer. Ginebra: Organización Internacional del Trabajo

OIT (2015). La Mujer en la gestión empresarial. Cobrando impulso. Ginebra: Organización Internacional del Trabajo

ONU-Mujeres (2016). Principios para el empoderamiento de las mujeres en las empresas. Mexico: Naciones Unidas

Panagiotakopoulos, A. (2020). Exploring the link between management training and organizational performance in the small business context. Journal of Workplace Learning, ahead-of-p(ahead-of-print). https://doi.org/10.1108/JWL-10-2019-0121

Pérez-Pérez, C., \& Avilés-Hernández, M. (2016). Explanatory factors of female entrepreneurship and limiting elements. Suma de Negocios, 7(15), 25-31. https://doi.org/10.1016/j.sumneg.2015.12.004

Quevedo Batista, E. P., Ruiz Lopez, C. A., Antonio Vidaña, P. R., \& Muñoz Palacios, L. C. (2017). Análisis de la cultura de capacitación en PYMES : Enfocado al centro del estado de Veracruz. Tecnológico del Centro de Veracruz

SELA (2010). Desarrollando Mujeres Empresarias: La Necesidad de Replantear Políticas y Programas de Género en el Desarrollo de PYMES. Secretaría Permanente del SELA, 5, 55

SENCE (2013). Barreras para la capacitación de mujeres en cursos tradicionalmente masculinos. Santiago de Chile: Secretaría de Trabajo y Previsión Social, Servicio Nacional de Capacitación y Empleo

SEP (2019). Principales cifras del sector educativo nacional 2018-2019. Ciudad de México: Secretaría de Educación Pública

Saavedra García, M. L., \& Camarena Adame, M. E. (2015). Diferencias en la Competitividad de las Empresas según el género del director. Neumann Business Review, 1(2), 70-86.

https://doi.org/10.22451/3002.nbr2015.vol1.1.8006 
Saavedra García, M. L., \& Camarena Adame, M. E. (2015). Retos para el emprendimiento femenino en américa latina. Criterio Libre, 13(22), 129-152.

https://doi.org/10.18041/1900-0642/criteriolibre.2015v13n22.129

Santhi, A. B. (2017). Women entrepreneurship: problem and prospects: a study of chittoor district. International Journal of Research in Commerce \& Management, 8(6), 6-12

Sapién Aguilar, A. L., Piñón Howlet, L. C., \& Gutiérrez Díez, M. C. (2014). Capacitación en la empresa mexicana: un estudio de formación en el trabajo. Civilizar, 14(27), 123. https://doi.org/10.22518/16578953.185

Secretaria de Economía (2009). Acuerdo para la estratificación empresarial. México: Diario oficial de la federación, segunda sección, 30 de junio

Todaro, R., Godoy, L., \& Abramo, L. (2002). Desempeño laboral de hombres y mujeres: opinan los empresarios. Cadernos Pagu, 17-18, 197-236. https://doi.org/10.1590/S0104-83332002000100008

Tonoyan, V., Strohmeyer, R., \& Jennings, J. E. (2020). Gender Gaps in Perceived Start-up Ease: Implications of Sex-based Labor Market Segregation for Entrepreneurship across 22 European Countries. Administrative Science Quarterly, 65(1), 181-225. https://doi.org/10.1177/0001839219835867

Tripathi, K. A., \& Singh, S. (2018). Analysis of barriers to women entrepreneurship through ISM and MICMAC. Journal of Enterprising Communities: People and Places in the Global Economy, 12(3), 346-373. https://doi.org/10.1108/JEC-12-2017-0101

Tverdostup, M., \& Paas, T. (2017). Gender-specific human capital: identification and quantifying its wage effects. International Journal of Manpower, 38(6), 854-874. https://doi.org/10.1108/IJM-05-2016-0111

Valdivia, M. (2014). ¿Algo más que capacitación empresarial para el empoderamiento de mujeres microempresarias? Evidencia experimental de corto y mediano plazo en el Perú. Lima: GRADE. Documento de Investigación, 75. Retrieved from https://www.grade.org.pe/publicaciones/algo-mas-que-c apacitacion-empresarial-para-el-empoderamiento-de-mujeres-microempresarias-evidencia-experimentalde-corto-y-mediano-plazo-en-el-peru/

Value for Women (2018). Entendiendo cómo apoyar el potencial de las mujeres emprendedoras en México. Retrieved from https://v4w.org/wp-content/uploads/2019/09/Entendiendo-como-apoyar-el-potencial-de-mujeresemprendedoras-Español.pdf

Varadarajan Sowmya, D., Majumdar, S., \& Gallant, M. (2010). Relevance of education for potential entrepreneurs: an international investigation. Journal of Small Business and Enterprise Development, 17(4), 626-640. https://doi.org/10.1108/14626001011088769

Welsh, D. H. B., Kaciak, E., \& Shamah, R. (2018). Determinants of women entrepreneurs' firm performance in a hostile environment. Journal of Business Research, 88, 481-491. https://doi.org/10.1016/j.jbusres.2017.12.015

Zabludovsky, G. (2001). Empresarias y ejecutivas en México y Brasil. Mexico: Miguel Angel Porrúa Grupo Editorial 